\title{
Convergence to stable laws for multidimensional stochastic recursions: the case of regular matrices
}

\author{
Ewa Damek, Sebastian Mentemeier†, Mariusz Mirek*, Jacek Zienkiewicz*
}

November 15, 2018

Given a sequence $\left(M_{n}, Q_{n}\right)_{n \geq 1}$ of i.i.d. random variables with generic copy $(M, Q) \in$ $G L(d, \mathbb{R}) \times \mathbb{R}^{d}$, we consider the random difference equation (RDE)

$$
R_{n}=M_{n} R_{n-1}+Q_{n},
$$

$n \geq 1$, and assume the existence of $\kappa>0$ such that

$$
\lim _{n \rightarrow \infty}\left(\mathbb{E}\left\|M_{1} \cdots M_{n}\right\|^{\kappa}\right)^{\frac{1}{n}}=1 .
$$

We prove, under suitable assumptions, that the sequence $S_{n}=R_{1}+\cdots+R_{n}$, appropriately normalized, converges in law to a multidimensional stable distribution with index $\kappa$. As a by-product, we show that the unique stationary solution $R$ of the RDE is regularly varying with index $\kappa$, and give a precise description of its tail measure.

Keywords: Weak limit theorems, random difference equations, stable laws, stochastic recursions, multivariate regular variation.

AMS 2010 Subject classification: Primary 60F05; secondary 60J05, 60E07, $60 \mathrm{H} 25$.

\section{Introduction}

Let $\left(M_{n}, Q_{n}\right)_{n \geq 1}$ be a sequence of i.i.d. random variables with generic copy $(M, Q)$ such that $M$ is a real $d \times d$ matrix and $Q$ takes values in $\mathbb{R}^{d}$. Suppose further that

$$
\mathbb{E} \log ^{+}\|M\|<\infty
$$

\footnotetext{
${ }^{*}$ University of Wroclaw, Institute of Mathematics, Grunwaldzki 2/4, 50-384 Wroclaw. E. Damek was partially supported by MNiSW grant N N201 393937, e-mail: edamek@math.uni.wroc.pl, M. Mirek was partially supported by MNiSW grant N N201 392337, e-mail: mirek@math.uni.wroc.pl, J. Zienkiewicz was partially supported by MNiSW grant N N201 397137, e-mail zenek@math.uni.wroc.pl.

${ }^{\dagger}$ University of Muenster, Institut für Mathematische Statistik, Einsteinstraße 62, 48149 Münster. Research supported by the Deutsche Forschungsgemeinschaft (SFB 878). e-mail: mentemeier@uni-muenster.de
} 
where $\|M\|:=\sup _{|x|=1}|x M|$. Then, with $\Pi_{n}:=M_{1} \cdot \ldots \cdot M_{n}$, there exists $\beta \in[-\infty, \infty)$ such that

$$
\beta:=\lim _{n \rightarrow \infty} n^{-1} \log \left\|\Pi_{n}\right\| \quad \mathbb{P} \text {-a.s. }
$$

and defines the Liapunov exponent of the RDE

$$
R_{n}=M_{n} R_{n-1}+Q_{n}, \quad n \geq 1 .
$$

If $\beta$ is negative and

$$
\mathbb{E} \log ^{+}\|Q\|<\infty
$$

then this recursive Markov chain has a unique stationary distribution which is given by the law of the almost surely convergent series

$$
R:=\sum_{n \geq 1} \Pi_{n-1} Q_{n}
$$

This by now standard result may easily be deduced from a more general one for iterations of random Lipschitz maps, see e.g. [5] or [12] and references given there. Notice that $R$ can also be characterised as the unique solution to the associated stochastic fixed point equation (SFPE),

$$
R \stackrel{d}{=} M R+Q
$$

where $\stackrel{d}{=}$ denotes equality in distribution.

Asymptotic properties of $R$ and $R_{n}$ were first investigated by Kesten in his seminal paper [21], and they are of interest in various fields, e.g. financial time series or ARCH processes that can be considered as a special case of (10), see [10], 22]. Under suitable assumptions, the asymptotic behavior of $R$ is governed by the value $\kappa>0$, which is the unique positive solution to the equation

$$
\lim _{n \rightarrow \infty}\left(\mathbb{E}\left\|\Pi_{n}\right\|^{\kappa}\right)^{\frac{1}{n}}=1 .
$$

If $M$ is restricted to the set of similarities, this condition simplifies to $\mathbb{E}\|M\|^{\kappa}=1$. Then, $\mathrm{Bu}$ raczweski et al. showed that $R$ is regular varying [ $\left[\right.$, and that the sequence $S_{n}:=R_{1}+\cdots+R_{n}$, properly normalized, converges in law to a multidimensional stable distribution with index $\kappa$ [6]. See also [25] for a generalization to Lipschitz recursions.

In the present article, we apply these methods, which were developed by Guivarc'h and Le Page in [15], to the case where $M_{n}$ takes values in $G L(d, R)$, and its distribution powers satisfy some density and irreducibility assumptions (stated below) which were considered in [1]. The major ingredient is a theorem by Basrak et al. 2] that links regular variation of $R$ with regular variation of its components $\langle x, R\rangle$. For related results on the tail behavior of solutions to (1) under varying assumptions on the distribution of $M$, see [11],[16], [24], as well as [14] for the case $d=1$.

In Section 2 we review the results of [1] and [2]. Then we state our main results in Section 3 . The proofs are given in Sections 4 to 6 ,

After submission of this paper, we learned about the recent preprint by Gao et al. [13]. They use more intricate analysis to give similar results under more general conditions and in the case $\kappa=2$. 


\section{Prior results}

The assumptions on $(M, Q)$ considered here were first stated by Kesten [21, Theorem 6]. The proof of the regular behavior of $R$ (in a more general setting) was given by Le Page [24], see also the recent preprint [16]. Using Kesten's assumptions, a short proof was recently given by Alsmeyer and the second author by use of a different Markov Renewal Theorem [1]. Here we continue their investigations.

We start with recalling the main result of [1]. Given $x \in V:=\mathbb{R}^{d} \backslash\{0\}$, we write $x^{\sim}$ for its projection on the unit sphere $S:=S^{d-1}$, thus $x^{\sim}:=|x|^{-1} x$. We will consider both column and row vectors, without special mention as long as this is clear from the context. Only scalar products are always understood to be between column vectors. The Lebesgue measure on the space of real $d \times d$-matrices, seen as $\mathbb{R}^{d^{2}}$, is denoted as $\lambda^{d^{2}}$ and the Lebesgue measure on $\mathbb{R}_{+}$ as $\boldsymbol{\lambda}$. Finally, the open $\delta$-balls in $S$ and $G L(d, \mathbb{R})$ with centers $x$ and $A$ are denoted as $B_{\delta}(x)$ and $B_{\delta}(A)$, respectively.

Theorem 2.1 (Theorem 1.1 in [1]).

Consider the RDE (11) and suppose that, in addition to (A1), (A2) and $\beta<0$, the following assumptions hold:

$$
\begin{aligned}
& \mathbb{P}(M \in G L(d, \mathbb{R}))=1 . \\
& \max _{n \geq 1} \mathbb{P}\left(\left(v \Pi_{n}\right)^{\sim} \in U\right)>0 \text { for any } v \in S \text { and any open } \emptyset \neq U \subset S . \\
& \mathbb{P}\left(\Pi_{n_{0}} \in \cdot\right) \geq \gamma_{0} \mathbf{1}_{B_{c}\left(\Gamma_{0}\right)} \mathbb{d}^{d^{2}} \text { for some } \Gamma_{0} \in G L(d, \mathbb{R}), n_{0} \in \mathbb{N} \text { and } c, \gamma_{0}>0 . \\
& \mathbb{P}(M r+Q=r)<1 \text { for any column vector } r \in \mathbb{R}^{d} . \\
& \text { There exists } \kappa_{0}>0 \text { such that } \\
& \quad \mathbb{E} \inf _{v \in S}|v M|^{\kappa_{0}} \geq 1, \mathbb{E}\|M\|^{\kappa_{0}} \log ^{+}\|M\|<\infty \text { and } 0<\mathbb{E}\|Q\|^{\kappa_{0}}<\infty .
\end{aligned}
$$

Then there exists a unique $\kappa \in\left(0, \kappa_{0}\right]$ such that

$$
\varrho(\kappa):=\lim _{n \rightarrow \infty}\left(\mathbb{E}\left\|\Pi_{n}\right\|^{\kappa}\right)^{\frac{1}{n}}=1,
$$

and

$$
\lim _{t \rightarrow 0} t^{-\kappa} \mathbb{P}(v(t R)>1)=K(v) \quad \text { for all } v \in S,
$$

where $K$ is a finite positive and continuous function on $S$.

Remark 2.2. Assumption (A4) holds in particular if $\Gamma_{0}=I d$ in (A5). If $M$ and $Q$ are independent, then (A6) holds trivially. Regarding the condition $\mathbb{E} \inf _{v \in S}|v M|^{\kappa_{0}}$, it is shown in [1, Section 5.1] that the function $\varrho(\varkappa)$ is log-convex and $\varrho(\delta)<1$ for some $\delta>0$. Then this condition asserts that at least $\varrho\left(\kappa_{0}\right) \geq 1$, and so it implies the existence of $\kappa$. This is the only place it is needed and so it may be replaced by any other condition assuring the existence of $\kappa_{0}$ such that $\varrho\left(\kappa_{0}\right) \geq 1$, compare [22, Remark 2.8 (iii)]. Our theorem is then applicable to the situation of [22].

Next, we note an explicit description of the function $K(x)$. Therefore, we introduce the operator on continuous functions on the sphere

$$
T_{\kappa}: \mathcal{C}(S) \rightarrow \mathcal{C}(S), \quad T_{\kappa} f(v):=\mathbb{E}\left(f\left((v M)^{\sim}\right)|v M|^{\kappa}\right),
$$


which was studied in [1, Section 5.1]. The operator $T_{\kappa}$ is quasi-compact, its spectral radius is given by $\varrho(\kappa)=1$, the only eigenvalue with modulus one is 1 , and the corresponding eigenspace is one-dimensional.

Proposition 2.3. The function $K(v)$ in (51) is given by

$$
0<K(v)=\frac{r(v)}{\alpha \kappa} \int_{S} \frac{1}{r(y)} \mathbb{E}\left(\left((y R)^{+}\right)^{\kappa}-\left((y M R)^{+}\right)^{\kappa}\right) \pi(d y)<\infty .
$$

If $\eta$ is the unique distribution such that $\eta T_{\kappa}=\eta$, then $r$ is the unique function with the property $T_{\kappa} r=r$ and $\int_{S} r(v) \eta(d v)=1$. Finally, the distribution $\pi$ is given by $\pi(d v)=r(v) \eta(d v)$, and

$$
\alpha=\int_{S} \frac{1}{r(y)} \mathbb{E}\left(\log |y M| r\left((y M)^{\sim}\right)|y M|^{\kappa}\right) \pi(d y) .
$$

Proof. The formula for $K(v)$ is given in [1, Lemmas 6.1 and 6.4]. The function $r$ is defined by $T_{\kappa} r=r$ in [1, Lemma 5.4]. Then $\pi$ is defined as the unique stationary distribution of the Markov chain with transition kernel ${ }^{\kappa} P$ given by (see [1, (18)]) ${ }^{\kappa} P f(v):=r(v)^{-1} T_{\kappa}(f \cdot r)(v)$. Thus

$$
\int_{S}(f(v) \cdot r(v)) \frac{\pi(d v)}{r(v)}=\int_{S} T_{\kappa}(f \cdot r) \frac{\pi(d v)}{r(v)} .
$$

Since $r$ is continuous and positive, any function $g \in \mathcal{C}(S)$ can be written as $g=f \cdot r, f \in \mathcal{C}(S)$, thus $\left(r^{-1} \pi\right) T_{\kappa}=\left(r^{-1} \pi\right)$, and by uniqueness, $\pi=r \eta$. The expression for $\alpha$ may be found in [1, Lemma 5.9].

The random variable $R$ is said to be regularly varying with index $\kappa$, if there exists a slowly varying function $L$ on $\mathbb{R}_{+}$and a Radon measure $\Lambda_{\kappa}$ on $V$ (called tail measure), such that for all compactly supported continuous $f$ (i.e. $f \in \mathcal{C}_{c}(V)$ )

$$
\lim _{t \rightarrow 0} t^{-\kappa} L\left(t^{-1}\right) \mathbb{E}(f(t R))=\int_{V} f(x) \Lambda_{\kappa}(d x) .
$$

Basrak et. al. [2] investigated conditions under which (5) already implies that $R$ is regularly varying with index $\kappa$. For noninteger $\kappa$, this holds true, see [2, Theorem 1.1 (ii)]. If $\kappa$ is an odd integer, a close inspection of their proof shows that it still goes through, provided the distribution of $R$ is symmetric, i.e. $\mathbb{P}(R \in \cdot)=\mathbb{P}(-R \in \cdot)$. This can also be deduced from [4, Corollary 2] combined with a comment after the proof of [4, Theorem 3b]. Both [2], 4] also give counterexamples, showing that the condition $\kappa \notin \mathbb{N}$ in general may not be omitted.

For future reference, we formulate this result as a proposition (see also [23, Theorem 4.3]). Note that by (2), a sufficient condition for $R$ to have a symmetric distribution is that $\mathrm{Q}$ has a symmetric distribution.

Proposition 2.4. Under the assumptions of Theorem [2.1, if

- $\kappa \notin \mathbb{N}$, or

- $\kappa$ odd, and $Q$ has symmetric distribution,

then $R$ is regularly varying with index $\kappa$. The function $L$ is equal to $\mathbf{1}$. 


\section{Statement of results}

Our first theorem gives more precise information about the tail measure, and extends the class of test functions in (8). Its proof will be given in section [4. By $T_{\kappa}^{*}$ we denote the operator

$$
T_{\kappa}^{*}: \mathcal{C}(S) \rightarrow \mathcal{C}(S), \quad T_{\kappa}^{*} f(v):=\mathbb{E}\left(f\left((M v)^{\sim}\right)|M v|^{\kappa}\right) .
$$

Theorem 3.1. Under the assumptions of [2.1, let $\kappa \notin \mathbb{N}$ or $\kappa$ odd and $Q$ symmetric. Then the tail measure $\Lambda_{\kappa}$ is a product measure $\Lambda_{\kappa}=\sigma_{\kappa} \otimes \mathbb{A}_{\kappa}$ on $V=\mathbb{R}^{d} \backslash\{0\}$, where $\sigma_{\kappa}$ is a finite nonzero measure satisfying $\sigma_{\kappa} T_{\kappa}^{*}=\sigma_{\kappa}$, and $\mathbb{A}_{\kappa}(d s):=s^{-(\kappa+1)} \mathbb{\lambda}(d s)$. Moreover, for every $\Lambda_{\kappa}$-a.e. continuous function $f$, such that

$$
\sup _{x \in V}|x|^{-\kappa}|\log | x||^{1+\varepsilon}|f(x)|<\infty
$$

for some $\varepsilon>0$, we have

$$
\lim _{t \rightarrow 0} t^{-\kappa} \mathbb{E}(f(t R))=\int_{V} f(x) \Lambda_{\kappa}(d x)\left(=\int_{0}^{\infty} \int_{S} f(s v) \sigma_{\kappa}(d v) \frac{1}{s^{\kappa+1}} d s\right) .
$$

Remark 3.2. It is interesting to compare this result with [9, Theorem 1.10], which describes the tail measure in the case where the heavy-tail behaviour of $R$ is caused by heavy-tailed input $Q$.

A distribution on $\mathbb{R}^{d}$ with characteristic function $\Xi$ is called stable, if for all $n \in \mathbb{N}$ there exist $\gamma_{n}>0$ and $x_{n} \in \mathbb{R}^{d}$, such that for all $y \in \mathbb{R}^{d}$

$$
(\Xi(y))^{n}=\Xi\left(\gamma_{n} y\right) e^{i\left\langle x_{n}, y\right\rangle} .
$$

This holds in particular true, if there is $\kappa>0$ and a function $C_{\kappa}: S \rightarrow \mathbb{C}$, such that for all $s \geq 0, v \in S$,

$$
\Xi(s v)=\exp \left(s^{\kappa} C_{\kappa}(v)\right)
$$

since then

$$
(\Xi(s v))^{n}=\exp \left(n s^{\kappa} C_{\kappa}(v)\right)=\exp \left(\left(n^{\frac{1}{\kappa}} s\right)^{\kappa} C_{\kappa}(v)\right)=\Xi\left(\left(n^{\frac{1}{\kappa}} s\right) v\right) .
$$

A distribution on $\mathbb{R}^{d}$ is fully nondegenerate, i.e. its support is not contained in any lower dimensional (=proper) subspace of $\mathbb{R}^{d}$ iff the set $\left\{y \in \mathbb{R}^{d}:|\Xi(y)|<1\right\}$ is not contained in a proper subspace. This can easily be seen by considering all onedimensional marginal distributions. If $\Xi$ is of the form (13), then an equivalent condition is that the set $\{v \in S$ : $\left.\Re C_{\kappa}(v)<0\right\}$ is not contained in any proper subspace of $\mathbb{R}^{d}$.

Now we are ready to formulate our main theorem, which will be proved in sections 5 and 6 ,

Theorem 3.3. Under the assumptions of Theorem 2.1, write $R_{n}^{x}$ for the $n$-th iteration of (11) started with $R_{0}=x$, and $S_{n}^{x}:=\sum_{k=1}^{n} R_{k}^{x}$. Let $W(x)=\sum_{k=1}^{\infty} M_{k} \cdot \ldots \cdot M_{1} x$, and $h_{v}(x)=$ $\mathbb{E}\left(e^{i\langle v, W(x)\rangle}\right)$ for $x \in \mathbb{R}^{d}$ and $v \in S$. If $\kappa=1$, assume that the distribution of $Q$ is symmetric and set $\xi(t)=\mathbb{E}\left(\frac{t R}{1+|t R|^{2}}\right)$. 
- If $\kappa \in(0,1) \cup(1,2)$, then there is a sequence $d_{n}=d_{n}(\kappa)=n m_{\kappa}$ and a function $C_{\kappa}: S \mapsto \mathbb{C}$ such that the random variables $n^{-\frac{1}{\kappa}}\left(S_{n}^{x}-d_{n}\right)$ converge in law to the $\kappa$-stable random variable with characteristic function of the form (13), where

$$
C_{\kappa}(v)=\int_{V}\left(\left(e^{i\langle v, x\rangle}-1\right) h_{v}(x)-i \mathbf{1}_{(1,2)}(\kappa)\langle v, x\rangle\right) \Lambda_{\kappa}(d x),
$$

and $m_{\kappa}=\mathbf{1}_{(1,2)}(\kappa) \mathbb{E} R$.

- If $\kappa=1$, then there is a function $C_{1}: S \mapsto \mathbb{C}$ such that the random variables $n^{-1} S_{n}^{x}-$ $n \xi\left(n^{-1}\right)$ converge in law to the 1-stable distribution with characteristic function of the form (13), where

$$
C_{1}(v)=\int_{V}\left(\left(e^{i\langle v, x\rangle}-1\right) h_{v}(x)-\frac{i\langle v, x\rangle}{1+|x|^{2}}\right) \Lambda_{1}(d x) .
$$

Moreover, if

$$
\max _{n \geq 1} \mathbb{P}\left(\left(v M_{1}^{\top} \cdots M_{n}^{\top}\right)^{\sim} \in U\right)>0 \text { for any } v \in S \text { and any open } \emptyset \neq U \subset S,
$$

holds, then the limit laws are fully nondegenerate.

The case where $\kappa>2$ has been widely investigated in the context of Lipschitz maps defined on complete separable metric spaces, see [3, 28, where the authors exploited martingale methods. On the other hand we refer to [17, 18, 19] where, like in our case, the spectral methods play crucial roles.

Remark 3.4. Note that (A4) is the only assumption that changes when considering the transpose $M^{\top}$ instead of $M$. So using (A4*) instead of (A4), Theorem 2.1 holds for the solution $R^{*}$ of the SFPE

$$
R^{*} \stackrel{d}{=} M^{\top} R^{*}+Q
$$

with the same $\kappa$ due to (4).

Remark 3.5. Similar results can be obtained in the case of nonnegative $(M, Q)$, satisfying the assumptions of Kesten [21, Theorem B] - see also [7, 16, 26] for weaker versions of these assumptions. The proof goes along the same lines, just starting with [21, Theorem B] instead of our Theorem 2.1. The limit laws are supported in the positive cone if $\kappa<1$.

\section{On the tail measure}

Proposition 2.4 yields the regular variation of $R$, given $\kappa \notin \mathbb{N}$, or $\kappa$ odd, and $Q$ having symmetric distribution. Property (8) implies that $\Lambda_{\kappa}$ is a product measure on $V$ considered as $S \times \mathbb{R}_{+}$. We will show that

$$
\int_{V} f(x) \Lambda_{\kappa}(d x)=\int_{0}^{\infty} \int_{S} f(r x) \sigma_{\kappa}(d x) \frac{d r}{r^{\kappa+1}}
$$


Indeed, let $\Phi: V \mapsto(0, \infty) \times S$ be defined as follows: $\Phi(x)=\left(|x|, \frac{x}{|x|}\right)$ and its inverse $\Phi^{-1}:(0, \infty) \times S \mapsto V$ by $\Phi^{-1}(r, z)=r z$. Let us define the measure $\sigma_{\kappa}$ on $S$

$$
\sigma_{\kappa}(A):=\kappa \Lambda_{\kappa}\left(\Phi^{-1}[(1, \infty) \times A]\right),
$$

for $A \in \mathcal{S}$. Now on the one hand we have that

$$
\int_{V} f(x) \Lambda_{\kappa}(d x)=\int_{(0, \infty) \times S} f(r z)\left(\Lambda_{\kappa} \circ \Phi^{-1}\right)(d r, d z) .
$$

On the other hand for all $A \in \mathcal{S}$ and $s>0$ we have

$$
\begin{aligned}
& \Lambda_{\kappa}\left(\Phi^{-1}[A \times(s, \infty)]\right)=\Lambda_{\kappa}\left(s \Phi^{-1}[A \times(1, \infty)]\right) \\
&=\lim _{t \rightarrow 0} t^{-\kappa} \mathbb{P}\left(t R \in s \Phi^{-1}[A \times(1, \infty)]\right)=s^{-\kappa} \lim _{t \rightarrow 0}\left(\frac{t}{s}\right)^{-\kappa} \mathbb{P}\left(\frac{t}{s} R \in \Phi^{-1}[A \times(1, \infty)]\right) \\
& \quad=s^{-\kappa} \Lambda_{\kappa}\left(\Phi^{-1}[A \times(1, \infty)]\right)=\kappa \int_{s}^{\infty} \Lambda_{\kappa}\left(\Phi^{-1}[A \times(1, \infty)]\right) t^{-\kappa-1} d t .
\end{aligned}
$$

Thus $\Lambda_{\kappa}=\sigma_{\kappa} \otimes \mathbb{A}_{\kappa}$, and formula (15) follows.

Lemma 4.1. The measure $\sigma_{\kappa}$, as defined above, is finite and nonzero.

Proof. The measure $\sigma_{\kappa}$ is nonzero, since by (15)

$$
\begin{aligned}
\sigma_{\kappa}(S)=\kappa \Lambda_{\kappa}((1, \infty)) & =\lim _{t \rightarrow 0} t^{-\kappa} \mathbb{P}(|t R| \in(1, \infty)) \\
& \geq \lim _{t \rightarrow 0} t^{-\kappa} \mathbb{P}(\langle x, t R\rangle \in(1, \infty))=K(x)>0
\end{aligned}
$$

for all $x \in S$. On the other hand, observe that for $e_{1}, \ldots, e_{d}$ being an orthonormal basis of $\mathbb{R}^{d}$,

$$
t^{-\kappa} \mathbb{P}(|t R|>1) \leq \sum_{i=1}^{d}\left(t^{-\kappa} \mathbb{P}\left(\left\langle e_{i}, t R\right\rangle>d^{-2}\right)+t^{-\kappa} \mathbb{P}\left(\left\langle-e_{i}, t R\right\rangle>d^{-2}\right)\right),
$$

and the limit of the right hand side is finite again by (15).

Remark 4.2. This shows in particular, that for all $\varepsilon>0$ there exists $C>0$ such that

$$
\Lambda_{\kappa}(S \times[C, \infty))=\Lambda_{\kappa}\left(B_{C}(0)^{c}\right)<\varepsilon .
$$

This is the main ingredient for showing that (11) holds for the more general class of test functions given in (10). The proof goes along the same lines as the proof of Theorem 2.8 in [8] and is therefore omitted.

The next proposition characterizes $\Lambda_{\kappa}$ as a stationary measure of the Markov Chain on $V$, given by the action of $M$ on $V$.

Proposition 4.3. For the tail measure $\Lambda_{\kappa}$, and all $f \in \mathcal{C}_{c}(V)$,

$$
\int_{V} f(x) \Lambda_{\kappa}(d x)=\int_{V} \mathbb{E}(f(M x)) \Lambda_{\kappa}(d x) .
$$


Proof. We introduce the space of $\varepsilon$-Hölder continuous functions, defined by

$$
H^{\varepsilon}=\left\{f \in \mathcal{C}_{c}(V): \sup _{x, y \in V} \frac{|f(x)-f(y)|}{|x-y|^{\varepsilon}}<\infty\right\} .
$$

One can proceed as in the proof of Lemma 2.19 in [8], to show that if $0<\varepsilon<\min \{1, \kappa\}$, then for all $f \in H^{\varepsilon}$

$$
\lim _{t \rightarrow 0} t^{-\kappa} \mathbb{E}(f(t R)-f(t M R))=0 .
$$

Since $\lim _{t \rightarrow 0} t^{-\kappa} \mathbb{E}(f(t R))$ exists, this yields the existence of $\lim _{t \rightarrow 0} t^{-\kappa} \mathbb{E}(f(t M R))$, and it is equal to $\int_{V} f(x) \Lambda_{\kappa}(d x)$. It remains to show that the limit is also equal to $\int_{V} \mathbb{E}(f(M x)) \Lambda_{\kappa}(d x)$ - note that $x \mapsto \mathbb{E}(f(M x))$ in general has unbounded support. Let $\operatorname{supp} f \subset{\overline{B_{\eta}(0)}}^{c}, \eta>0$. Then for any $t>0$ and $m \in G L(\mathbb{R}, d)$,

$$
\begin{aligned}
& t^{-\kappa} \mathbb{E}(f(t m R)) \leq t^{-\kappa}\|f\|_{\infty} \mathbb{E}\left(\mathbf{1}_{\{|t m R|>\eta\}}\right) \\
& \leq\|m\|^{\kappa}\|f\|_{\infty}\left(t^{-\kappa}\|m\|^{-\kappa} \mathbb{P}(t\|m\||R|>\eta)\right) \\
& \leq\|m\|^{\kappa}\|f\|_{\infty} \sup _{s>0} s^{-\kappa} \mathbb{P}(s|R|>\eta),
\end{aligned}
$$

and as above, using (15), $C:=\sup _{s>0} s^{-\kappa} \mathbb{P}(s|R|>\eta)<\infty$, thus

$$
\int\left(\|m\|^{\kappa}\|f\|_{\infty} \sup _{s>0} s^{-\kappa} \mathbb{P}(s|R|>\eta)\right) \mathbb{P}(M \in d m)=C\|f\|_{\infty} \mathbb{E}\left(\|M\|^{\kappa}\right)<\infty .
$$

Then we may use the dominated convergence theorem and that for fixed $m \in G L(d, \mathbb{R})$, $x \mapsto f(m x)$ has compact support, to infer

$$
\begin{aligned}
& \lim _{t \rightarrow 0} t^{-\kappa} \mathbb{E}(f(t M R))=\int \lim _{t \rightarrow 0} t^{-\kappa} \mathbb{E}(f(t m R)) \mathbb{P}(M \in d m) \\
= & \iint_{V} f(m x) \Lambda_{\kappa}(d x) \mathbb{P}(M \in d m)=\int_{V} \mathbb{E}(f(M x)) \Lambda_{\kappa}(d x) .
\end{aligned}
$$

Finally observe that $H^{\varepsilon}$ is dense in $\mathcal{C}_{c}(V)$ due to the Stone-Weierstrass theorem, so we infer the assumption for all $f \in \mathcal{C}_{c}(V)$.

Lemma 4.4. For the operator $T_{\kappa}^{*}$ introduced in (9), $\sigma_{\kappa} T_{\kappa}^{*}=\sigma_{\kappa}$.

Proof. First notice that due to Remark 4.2, the identity (16) also holds for bounded continuous functions $f$ on $V$ such that $\operatorname{supp} f \cap B_{\eta}(0)=\emptyset$ for some $\eta>0$, in particular for functions $g_{u}(s v)=f(v) \mathbf{1}_{(u, \infty)}(s)$, where $f$ is any continuous function on $S$, and $u>0$. Then

$$
\begin{aligned}
& \int_{u}^{\infty} \int_{S} f(v) \sigma_{\kappa}(d v) \frac{1}{s^{\kappa+1}} d s=\int_{0}^{\infty} \int_{S} \mathbb{E}\left(f\left((M v)^{\sim}\right) \mathbf{1}_{(u, \infty)}(s|M v|)\right) \sigma_{\kappa}(d v) \frac{1}{s^{\kappa+1}} d s \\
= & \mathbb{E}\left(\int_{S} \int_{0}^{\infty} f\left((M v)^{\sim}\right) \mathbf{1}_{(u, \infty)}(t) \frac{|M v|^{\kappa}}{t^{\kappa+1}} d t \sigma_{\kappa}(d v)\right) \\
= & \int_{u}^{\infty} \int_{S} \mathbb{E}\left(f\left((M v)^{\sim}\right)|M v|^{\kappa}\right) \sigma_{\kappa}(d v) \frac{1}{t^{\kappa+1}} d t .
\end{aligned}
$$

Since $u$ is arbitrary, we infer $\int_{S} f(v) \sigma_{\kappa}(d v)=\int_{S} \mathbb{E}\left(f\left((M v)^{\sim}\right)|M v|^{\kappa}\right) \sigma_{\kappa}(d v)$ for all $f \in \mathcal{C}(S)$. 


\section{Convergence to stable laws}

The proof of Theorem 3.3 is based on the spectral method initiated by Nagaev [27] and its first application in this context due to Guivarc'h and Le Page [15]. For $t>0, v \in S$, we consider Fourier perturbations

$$
P_{t, v} f(x)=\mathbb{E}\left(e^{i\langle t v, M x+Q\rangle} f(M x+Q)\right)=\mathbb{E}\left(e^{i\left\langle t v, R_{1}^{x}\right\rangle} f\left(R_{1}^{x}\right)\right),
$$

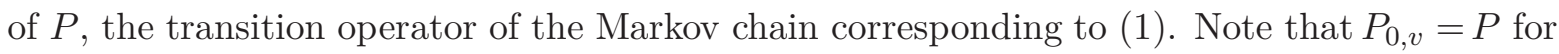
all $v \in S$. Since for $n \geq 1$,

$$
P_{t, v}^{n} f(x)=\mathbb{E}\left(e^{i\left\langle t v, S_{n}^{x}\right\rangle} f\left(R_{n}^{x}\right)\right),
$$

we are interested in the asymptotics of $P_{t, v}^{n} \mathbf{1}$.

The main tool used here is the Keller-Liverani theorem [20, which links the properties of $P$ and $P_{t, v}$ on appropriate function spaces for small $t$. The proof goes along the same lines as in [6], 25], so we only give an outline. In particular we do not check the assumptions of the theorem, which can be copied from [6]) for the case $\kappa \in(0,1) \cup(1,2)$. The case when $\kappa=1$ is more technical, the detailed exposition is contained in [9].

First observe that the unique eigenvalue of $P$, acting on $\mathcal{C}\left(\mathbb{R}^{d}\right)$, of modulus one is 1 , since the corresponding Markov chain has a unique stationary distribution $\nu=\mathbb{P}(R \in \cdot)$. On an appropriately chosen function space $\mathcal{B}$ containing $1, P$ also has a spectral gap, see [6, 3.13 and 3.14]. Thus $P$ is quasi-compact, i.e. we have the decomposition

$$
P=1 \cdot \Pi+Q
$$

where $\Pi$ is a one dimensional projection given by $\Pi f=\nu(f) \mathbf{1}$, the spectral radius of $Q$ is strictly smaller than 1, and both operators commute.

It is now a result of the Keller-Liverani-theorem that, for small t, the operators $P_{t, v}$, acting on $\mathcal{B}$, are also quasi-compact with singular dominant eigenvalue $k(t, v)$, such that for all $n \geq 1$

$$
P_{t, v}^{n}=k(t, v)^{n} \Pi_{t, v}+Q_{t, v}^{n},
$$

where again $\Pi_{t, v}$ is a onedimensional projection operator commuting with $Q_{t, v}$, and the spectral radii of $Q_{t, v}$ are uniformly bounded by some $\varrho<1$. Moreover, $k(t, v) \rightarrow 1$, as well as $\Pi_{t, v} \rightarrow \Pi$, $Q_{t, v} \rightarrow Q$ as operators on $\mathcal{B}$, see [6, 3.17 and 3.18]. The following lemma provides the link to the characteristic functions

$$
\Xi_{\kappa, n}(s v)=\mathbb{E}\left(e^{i\left\langle s v, n^{-1 / \kappa}\left(S_{n}^{x}-n m_{\kappa}\right)\right\rangle}\right)
$$

of the normalized Birkhoff sums.

Lemma 5.1. Under the assumptions of Theorem [3.3, with $t_{n}:=s n^{-1 / \kappa}$ for $n \in \mathbb{N}, s>0$ and $v \in S$ we have (provided the right hand side exists)

$$
\lim _{n \rightarrow \infty} \Xi_{\kappa, n}(s v)=\exp \left(s^{\kappa} \cdot \lim _{n \rightarrow \infty} \frac{k\left(t_{n}, v\right)-1-i\left\langle v, t_{n} m_{\kappa}\right\rangle}{t_{n}^{\kappa}}\right) .
$$


Proof. Using that $\lim _{s \rightarrow 0} \Pi_{s, v} \mathbf{1}(x)=1$ for all $x \in \mathbb{R}^{d}$ and $r\left(Q_{s, v}\right) \leq \varrho<1$, for all $v \in S$, and $s$ small enough, we see that $\lim _{n \rightarrow \infty} \Xi_{\kappa, n}(t v)$ equals

$$
\begin{aligned}
& \lim _{n \rightarrow \infty} \mathbb{E}\left(e^{i t_{n}\left\langle v, S_{n}^{x}-n m_{\kappa}\right\rangle}\right)=\lim _{n \rightarrow \infty} e^{-i n t_{n}\left\langle v, m_{\kappa}\right\rangle} \mathbb{E}\left(e^{i t_{n}\left\langle v, S_{n}^{x}\right\rangle}\right) \\
= & \lim _{n \rightarrow \infty} e^{-i n t_{n}\left\langle v, m_{\kappa}\right\rangle} \cdot\left(P_{t_{n}, v}^{n} \mathbf{1}\right)(x) \\
= & \lim _{n \rightarrow \infty} e^{-i n t_{n}\left\langle v, m_{\kappa}\right\rangle} \cdot\left(k^{n}\left(t_{n}, v\right)\left(\Pi_{t_{n}, v} \mathbf{1}\right)(x)+\left(Q_{t_{n}, v}^{n} \mathbf{1}\right)(x)\right) \\
= & \lim _{n \rightarrow \infty} e^{-i n t_{n}\left\langle v, m_{\kappa}\right\rangle} \cdot k^{n}\left(t_{n}, v\right) .
\end{aligned}
$$

Assume for the moment, that $n \cdot\left(e^{-i t_{n}\left\langle v, m_{\kappa}\right\rangle} \cdot k\left(t_{n}, v\right)-1\right)$ has a limit as $n$ goes to infinity (this will be shown later). We infer

$$
\begin{aligned}
\lim _{n \rightarrow \infty} e^{-i n t_{n}\left\langle v, m_{\kappa}\right\rangle} \cdot k^{n}\left(t_{n}, v\right) & =\lim _{n \rightarrow \infty}\left(1+\frac{1}{n} n \cdot\left(e^{-i t_{n}\left\langle v, m_{\kappa}\right\rangle} \cdot k\left(t_{n}, v\right)-1\right)\right)^{n} \\
& =\exp \left(\lim _{n \rightarrow \infty} n \cdot\left(e^{-i t_{n}\left\langle v, m_{\kappa}\right\rangle} \cdot k\left(t_{n}, v\right)-1\right)\right) \\
& =\exp \left(t^{\kappa} \cdot \lim _{n \rightarrow \infty} \frac{k\left(t_{n}, v\right)-1-i\left\langle v, t_{n} m_{\kappa}\right\rangle}{t_{n}^{\kappa}}\right) .
\end{aligned}
$$

The last line follows from the definition of $t_{n}$ and $m_{\kappa}=0$ if $\kappa \in(0,1)$. If $\kappa \in(1,2)$, we expand

$$
n \cdot\left(e^{-i t_{n}\left\langle v, m_{\kappa}\right\rangle} \cdot k\left(t_{n}, v\right)-1\right)=n \cdot\left(\left[1-i t_{n}\left\langle v, m_{\kappa}\right\rangle+o(1 / n)\right] \cdot\left[\left(k\left(t_{n}, v\right)-1\right)+1\right]-1\right) .
$$

In particular, if the limit in (17) exists, also the assumption made on the way is true, and our calculation was justified.

Thus we have to study the behaviour of $t^{-\kappa}\left(k(t, v)-1-i\left\langle v, t m_{\kappa}\right\rangle\right)$ as $t$ tends to zero.

Let $g_{t, v}$ be an eigenfunction of $P_{t, v}$, corresponding to the dominant eigenvalue $k(t, v)$. From $P_{t, v} g_{t, v}=P e^{i t\langle v, \cdot\rangle} g_{t, v}$ and $\nu P=\nu$ it follows that

$$
(k(t, v)-1) \nu\left(g_{t, v}\right)=\int_{\mathbb{R}^{d}}\left(e^{i t\langle v, x\rangle}-1\right) g_{t, v}(x) \nu(d x) .
$$

Now we need an explicit expression for $g_{t, v}$. It turns out that it is closely related to $h_{v}$ : Denote $\Delta_{t} f(x)=f(t x)$ for $t>0$. Introducing auxiliary operators

$$
T_{t, v} f(x)=\mathbb{E}\left(e^{i\langle v, M x+t Q\rangle} f(M x+t Q)\right)
$$

we have the identity

$$
T_{t, v}=\Delta_{t}^{-1} \circ P_{t, v} \circ \Delta_{t}
$$

This relates the eigenvalues of both operators for $t>0$. Namely, if $f$ is an eigenfunction of $T_{t, v}$, then $\Delta_{t} f$ is an eigenfunction of $P_{t, v}$. So it is not surprising that the Keller-Liverani-Theorem holds for the operators $T_{t, v}$, yielding a decomposition

$$
T_{t, v}=k(t, v) \Pi_{T, t, v}+Q_{T, t, v},
$$

with $k(t, v) \rightarrow k(0, v), \Pi_{T, t, v} \rightarrow \Pi_{T, 0, v}$. 
Now it is easy to check that $h_{v}(x)=\mathbb{E}\left(e^{i\left\langle v, \sum_{k=1}^{\infty} M_{k} \cdots M_{1} x\right\rangle}\right)$ is the eigenfunction of $T_{0, v}$ corresponding to eigenvalue $k(0, v)=1$, i.e. $\Pi_{T, 0, v} f=c_{f} \cdot h_{v}$. Since $\Pi_{T, t, v} h_{v} \neq 0$ for $t$ small enough and this is an eigenfunction of $T_{t} t, v$ corresponding to the eigenvalue $k(t, v)$, we have by (19) that

$$
\Delta_{t} \Pi_{T, t, v} h_{v}=c_{t} g_{t, v}
$$

for some $c_{t} \neq 0$. For more details we refer to [6] and [25].

Lemma 5.2. Under the assumptions of Theorem [3.3, for all $v \in S$, and $\kappa \in(0,1) \cup(1,2)$ we have

$$
\lim _{t \rightarrow 0} \frac{k(t, v)-1-i\left\langle v, t m_{\kappa}\right\rangle}{t^{\kappa}}=\int_{V}\left(\left(e^{i\langle v, x\rangle}-1\right) h_{v}(x)-i \mathbf{1}_{(1,2)}(\kappa)\langle v, x\rangle\right) \Lambda_{\kappa}(d x) .
$$

Proof. Using (201) in (18), yields

$$
(k(t, v)-1) \nu\left(\Delta_{t} \Pi_{T, t, v} h_{v}\right)=\int_{\mathbb{R}^{d}}\left(e^{i t\langle v, x\rangle}-1\right) \Delta_{t} \Pi_{T, t, v} h_{v}(x) \nu(d x) .
$$

Arguing in a similar way as in ([25, Section 6]), we obtain that

$$
\int_{\mathbb{R}^{d}}\left(e^{i t\langle v, x\rangle}-1\right) \cdot\left(\Delta_{t} \Pi_{T, t, v} h_{v}(x)-\Delta_{t} h_{v}(x)\right) \nu(d x)=o\left(t^{\kappa}\right)
$$

as well as $\left(\nu\left(\Delta_{t} \Pi_{T, t, v} h_{v}\right)-1\right)=o\left(t^{\kappa}\right)$. Thus

$$
\begin{aligned}
\lim _{t \rightarrow 0} \frac{k(t, v)-1}{t^{\kappa}} & =\lim _{t \rightarrow 0} t^{-\kappa} \int_{\mathbb{R}^{d}}\left(e^{i t\langle v, x\rangle}-1\right) \Delta_{t} h_{v}(x) \nu(d x) \\
& =\lim _{t \rightarrow 0} t^{-\kappa} \mathbb{E}\left(\left(e^{i\langle v, t R\rangle}-1\right) h_{v}(t R)\right) .
\end{aligned}
$$

If $\kappa \in(0,1)$, checking that $\left(e^{i\langle v, \cdot\rangle}-1\right) h_{v}$ satisfies (10) the proof follows. If $\kappa \in(1,2)$, we write

$$
\begin{aligned}
& \int_{\mathbb{R}^{d}}\left(e^{i t\langle v, x\rangle}-1\right) \Delta_{t} h_{v}(x) \nu(d x) \\
= & \int_{\mathbb{R}^{d}}\left(e^{i t\langle v, x\rangle}-1\right)\left(\Delta_{t} h_{v}(x)-1\right) \nu(d x)+\int_{\mathbb{R}^{d}}\left(e^{i t\langle v, x\rangle}-1-i\langle v, t x\rangle\right) \nu(d x)+i\left\langle v, t m_{\kappa}\right\rangle .
\end{aligned}
$$

We check that the functions under the integral satisfy (10) and then we use (23). For more details we refer to ([6, Section 5]).

This proves the pointwise convergence of $\Xi_{\kappa, n}(s v)$ to $\exp \left(s^{\kappa} C_{\kappa}(v)\right)$. Continuity at 0 follows from the dominated convergence theorem. 


\section{The limit measure is nondegenerate}

In this section we will assume additionally that assumption (A4*) is in force. As mentioned in Remark [3.4, all results of [1] then hold for the SFPE given by $\left(M^{\top}, Q\right)$.

We start with a corollary to Proposition 2.3. Note that $\sigma$ here is a measure on $S$ seen as set of column vectors.

Corollary 6.1. If $R$ is the solution to the $R D E R \stackrel{d}{=} M^{\top} R+Q$, then

$$
0<\int_{S} \mathbb{E}\left(\left(\langle R, y\rangle^{+}\right)^{\kappa}-\left(\left\langle M^{\top} R, y\right\rangle^{+}\right)^{\kappa}\right) \sigma(d y)<\infty
$$

where $\sigma$ is (up to scalar multiplication) the unique solution to $\sigma T_{\kappa}^{*}=\sigma$, with $T_{\kappa}^{*}$ as defined in (9).

Proof. Given ( $4^{*}$, Proposition 2.3 holds also for the RDE given by $\left(M^{\top}, Q\right)$, mutatis mutandis. Observe that considering $T_{\kappa}^{*}$ on functions of column vectors is equivalent to considering the operator $\hat{T}_{\kappa}$

$$
\hat{T}_{\kappa} f(v)=\mathbb{E}\left(f\left(\left(v M^{\top}\right)^{\sim}\right)\left|v M^{\top}\right|^{\kappa}\right)
$$

on functions of row vectors. But $\hat{T}_{\kappa}$ is the right expression for $T_{\kappa}$ of (6) for the RDE given by $\left(M^{\top}, Q\right)$. In particular the only eigenvalue of $T_{\kappa}^{*}$ of modulus one is 1 , and the corresponding eigenspace is one-dimensional. Finally use that $r$ is positive, and that $r(v)^{-1} \pi(d v)=\sigma(d v)$.

The following nice observation is the main ingredient in the proof of nondegeneracy:

Corollary 6.2. For $\sigma_{\kappa}$ as defined in Theorem [3.1, for $R$ being solution to $R \stackrel{d}{=} M^{\top} R+Q$,

$$
0<\int_{S} \mathbb{E}\left(\left(\left\langle M^{\top} R+Q, y\right\rangle^{+}\right)^{\kappa}-\left(\left\langle M^{\top} R, y\right\rangle^{+}\right)^{\kappa}\right) \sigma_{\kappa}(d y)<\infty
$$

Proof. Due to Theorem 3.1, $\sigma_{\kappa} T_{\kappa}^{*}=\sigma_{\kappa}$. But if A4* holds, this identifies $\sigma_{\kappa}$ up to scalar multiples, so $\sigma$ of Corollary 6.1 is a scalar multiple of $\sigma_{\kappa}$. One may replace $R$ by $M^{\top} R+Q$, since

$$
\mathbb{E}\left(\left(\langle R, y\rangle^{+}\right)^{\kappa}-\left(\left\langle M^{\top} R, y\right\rangle^{+}\right)^{\kappa}\right)=\int_{0}^{\infty} u^{\kappa-1}\left[\mathbb{P}(\langle R, y\rangle>u)-\mathbb{P}\left(\left\langle M^{\top} R, y\right\rangle>u\right)\right] d u,
$$

see [14, Lemma 9.4].

Lemma 6.3. Under the assumptions of Theorem 3.3 and with (A4* in force, the limit laws are fully nondegenerate, i.e. for all $v \in S^{\top} \Re C_{\kappa}(v)<0$.

Proof. Notice that

$$
\Re C_{\kappa}(v)=\Re\left(\int_{V}\left(e^{i\langle v, x\rangle}-1\right) \mathbb{E}\left[e^{i\langle v, W(x)\rangle}\right] \Lambda_{\kappa}(d x)\right)
$$




$$
\begin{aligned}
& =\int_{0}^{\infty} \int_{S} \mathbb{E}[\cos (s\langle v, W(w)+w\rangle)-\cos (s\langle v, W(w)\rangle)] \sigma_{\kappa}(d w) \frac{d s}{s^{\kappa+1}} \\
& =C(\kappa) \cdot \int_{S} \mathbb{E}\left[|\langle v, W(w)+w\rangle|^{\kappa}-|\langle v, W(w)\rangle|^{\kappa}\right] \sigma_{\kappa}(d w),
\end{aligned}
$$

for $C(\kappa)=\int_{0}^{\infty} \frac{\cos s-1}{s^{\kappa+1}}<0$. Let $W^{*} v:=\sum_{k=1}^{\infty} M_{1}^{\top} \cdots M_{k}^{\top} v$. Then it suffices to prove that

$$
\int_{S} \mathbb{E}\left[\left|\left\langle W^{*} v+v, w\right\rangle\right|^{\kappa}-\left|\left\langle W^{*} v, w\right\rangle\right|^{\kappa}\right] \sigma_{\kappa}(d w)
$$

is positive.

Therefore, we use that $\left(M^{\top}, v\right)$ satisifies the assumptions of Theorem 2.1, and that $W^{*} v+v$ is a solution of the random difference equation

$$
W^{*} v+v \stackrel{d}{=} M^{\top}\left(W^{*} v+v\right)+v
$$

In this situation, Corollary 6.2 yields

$$
0<\int_{S} \mathbb{E}\left(\left(\left\langle W^{*} v+v, w\right\rangle^{+}\right)^{\kappa}-\left(\left\langle W^{*} v, w\right\rangle^{+}\right)^{\kappa}\right) \sigma_{\kappa}(d w)<\infty
$$

and, considering $-v$ instead of $v$ (notice that $\sigma_{\kappa}$ does not depend on $v$ )

$$
0<\int_{S} \mathbb{E}\left(\left(\left\langle W^{*} v+v, w\right\rangle^{-}\right)^{\kappa}-\left(\left\langle W^{*} v, w\right\rangle^{-}\right)^{\kappa}\right) \sigma_{\kappa}(d w)<\infty
$$

Since both integrals are finite, adding (29) and (30) implies that (27) is indeed positive.

\section{ACKNOWLEDGEMENTS}

The major part of this work was done during two visits of the second author at the University of Wroclaw, Institute of Mathematics to which he wishes to express his gratitude for hospitality and a stimulating atmosphere.

The authors are grateful to the referee for a very careful reading of the manuscript and useful remarks that lead to the improvement of the presentation.

\section{References}

[1] G. Alsmeyer and S. Mentemeier. Tail behaviour of stationary solutions of random difference equations: the case of regular matrices. to appear in J. Difference Equ. Appl. DOI: 10.1080/10236198.2011.571383.

[2] B. Basrak, R. A. Davis, and T. Mikosch. A characterization of multivariate regular variation. Ann. Appl. Probab., 12(3):908-920, 2002.

[3] M. Benda. A central limit theorem for contractive stochastic dynamical systems. J. Appl. Probab., 35(1):200-205, 1998. 
[4] J. Boman and F. Lindskog. Support theorems for the Radon transform and Cramér-Wold theorems. J. Theoret. Probab., 22(3):683-710, 2009.

[5] P. Bougerol and N. Picard. Strict stationarity of generalized autoregressive processes. Ann. Probab., 20(4):1714-1730, 1992.

[6] D. Buraczewski, E. Damek, and Y. Guivarc'h. Convergence to stable laws for a class of multidimensional stochastic recursions. Probab. Theory Related Fields, 148:333-402, 2010.

[7] D. Buraczewski, E. Damek, and Y. Guivarc'h. On multidimensional Mandelbrot's cascades. ArXiv e-prints, Sept. 2011.

[8] D. Buraczewski, E. Damek, Y. Guivarc'h, A. Hulanicki, and R. Urban. Tail-homogeneity of stationary measures for some multidimensional stochastic recursions. Probab. Theory Related Fields, 145(3-4):385-420, 2009.

[9] D. Buraczewski, E. Damek, and M. Mirek. Asymptotics of stationary solutions of multivariate stochastic recursions with heavy tailed inputs and related limit theorems. Stochastic Process. Appl., 122(1):42 - 67, 2012.

[10] L. de Haan, S. I. Resnick, H. Rootzén, and C. G. de Vries. Extremal behaviour of solutions to a stochastic difference equation with applications to ARCH processes. Stochastic Process. Appl., 32(2):213-224, 1989.

[11] B. de Saporta, Y. Guivarc'h, and É. Le Page. On the multidimensional stochastic equation $Y_{n+1}=A_{n} Y_{n}+B_{n}$. C. R. Math. Acad. Sci. Paris, 339(7):499-502, 2004.

[12] P. Diaconis and D. Freedman. Iterated random functions. SIAM Review, 41:45-76, 1999.

[13] Z. Gao, Y. Guivarc'h, and E. Le Page. Spectral gap properties and convergence to stable laws for affine random walks on $\mathbb{R}^{d}$. ArXiv e-prints, Aug. 2011.

[14] C. M. Goldie. Implicit renewal theory and tails of solutions of random equations. Ann. Appl. Probab., 1(1):126-166, 1991.

[15] Y. Guivarc'h and E. Le Page. On spectral properties of a family of transfer operators and convergence to stable laws for affine random walks. Ergodic Theory Dynam. Systems, 28(2):423-446, 2008.

[16] Y. Guivarc'H and E. Le Page. Spectral gap properties and asymptotics of stationary measures for affine random walks. ArXiv e-prints, Apr. 2012.

[17] H. Hennion and L. Hervé. Limit theorems for Markov chains and stochastic properties of dynamical systems by quasi-compactness, volume 1766 of Lecture Notes in Mathematics. Springer-Verlag, Berlin, 2001.

[18] H. Hennion and L. Hervé. Central limit theorems for iterated random Lipschitz mappings. Ann. Probab., 32(3A):1934-1984, 2004.

[19] L. Hervé and F. Pène. The Nagaev-Guivarc'h method via the Keller-Liverani theorem. Bull. Soc. Math. France, 138(3):415-489, 2010.

[20] G. Keller and C. Liverani. Stability of the spectrum for transfer operators. Ann. Scuola Norm. Sup. Pisa Cl. Sci. (4), 28(1):141-152, 1999.

[21] H. Kesten. Random difference equations and renewal theory for products of random matrices. Acta Math., 131:207-248, 1973. 
[22] C. Klüppelberg and S. Pergamenchtchikov. The tail of the stationary distribution of a random coefficient AR(q) model. Ann. Appl. Probab., 14(2):971-1005, 2004.

[23] C. Klüppelberg and S. Pergamenchtchikov. Extremal behaviour of models with multivariate random recurrence representation. Stochastic Process. Appl., 117(4):432 - 456, 2007.

[24] É. Le Page. Théorèmes de renouvellement pour les produits de matrices aléatoires. Séminaires de probabilités Rennes. Publication des Séminaires de Mathématiques, Univ. Rennes I, pages 1-116, 1983.

[25] M. Mirek. Heavy tail phenomenon and convergence to stable laws for iterated lipschitz maps. Probab. Theory Related Fields, 151:705-734, 2011.

[26] M. Mirek. On fixed points of a generalized multidimensional affine recursion. ArXiv e-prints, Nov. 2011.

[27] S. V. Nagaev. Some limit theorems for stationary markov chains. Theory Probab. Appl., 2(4):378-406, 1957.

[28] W. B. Wu and M. Woodroofe. A central limit theorem for iterated random functions. $J$. Appl. Probab., 37(3):748-755, 2000. 